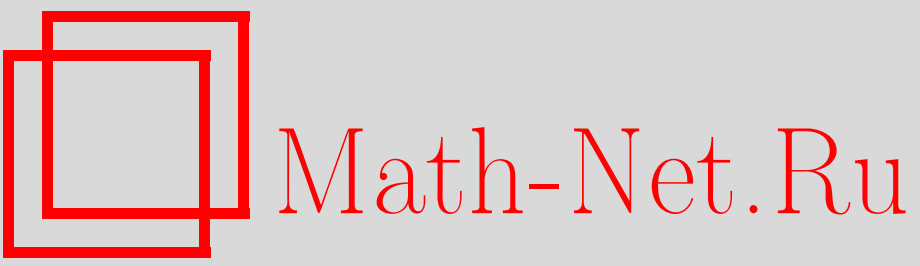

И. Д. Шкредов, Повторяемость неполных частных у цепных дробей, УМH, 2002, том 57, выпуск 4, 189-190

DOI: https://doi.org/10.4213/rm548

Использование Общероссийского математического портала Math-Net.Ru подразумевает, что вы прочитали и согласны с пользовательским соглашением

http://www.mathnet.ru/rus/agreement

Параметры загрузки:

IP : 35.174 .16 .151

26 апреля 2023 г., 14:59:57 


\title{
ПОВТОРЯЕМОСТЬ НЕПОЛНЫХ ЧАСТНЫХ У ЦЕПНЫХ ДРОБЕЙ
}

\author{
И. Д. ШКРЕДОВ
}

1. Формулировка основного результата. Цепной дробью, соответствующей числу $\alpha$, называется выражение $\alpha=a_{0}+\frac{1}{a_{1}+\frac{1}{a_{2}+\ldots}}=\left[a_{0} ; a_{1}, a_{2}, \ldots\right]$. Число $a_{n}$ назьвается $n$-м неполным частным числа $\alpha$, а рациональное число $p_{k} / q_{k}=\left[a_{0} ; a_{1}, a_{2}, \ldots, a_{k}\right]$ - подходящей дробью порядка $k$. Если $\alpha \in[0,1)$, то будем писать $\alpha=\left[a_{1}, a_{2}, \ldots\right]$. Все необходимые сведения о цепных дробях можно найти в $[1]$.

Рассмотрим множество $\Lambda_{M}$ цепных дробей с ограниченными неполными частными $a_{n} \leqslant M$ и зададим меру на нем следующим образом. Мера множества $\left\{\alpha \mid a_{n_{1}} \in A_{1}, \ldots, a_{n_{k}} \in A_{k}\right\}$, $A_{i} \subseteq\{1, \ldots, M\}$, равна $\prod\left|A_{i}\right| / M^{k}$, где $\left|A_{i}\right|$ означает количество элементов в $A_{i}$. Следующий метрический результат про повторяемость неполных частных сразу следует из теоремы о символьном потоке [2].

Для почти всех (относительно мерь, определенной выше) $\alpha=\left[a_{1}, a_{2}, \ldots\right] \in \Lambda_{M}$ существует возрастающая последовательность номеров $\left\{n_{\nu}\right\}_{\nu \in \mathbb{N}}$ такая, что

$$
a_{1}=a_{n_{\nu}+1}, \quad a_{2}=a_{n_{\nu}+2}, \ldots, \quad a_{k_{\nu}}=a_{n_{\nu}+k_{\nu}}
$$

$u k_{\nu} \geqslant \log _{M} n_{\nu} . C$ другой стороны, для любого $\varepsilon>0$ мера тех $\alpha \in \Lambda_{M}$, для которых существует возрастающая последовательность $\left\{n_{\nu}\right\}_{\nu \in \mathbb{N}}$ такая, что выполнено (1) $u$ $k_{\nu} \geqslant(1+\varepsilon) \log _{M} n_{\nu}$, равна нулю.

Сформулируем теперь наш основной результат.

ТеОРема. 1) Для любого $\varepsilon>0$ и для почти всех (относительно меры Лебега) чисел $\alpha$ существует возрастающая последовательность номеров $\left\{n_{\nu}\right\}_{\nu \in \mathbb{N}}$ такая, что выполнено (1) и $k_{\nu} \geqslant\left(6 \ln 2 / \pi^{2}-\varepsilon\right) \cdot \ln n_{\nu}$. 2) Для любого $\delta>0$ мера тех $\alpha$, для которых существует возрастающая последовательность $\left\{n_{\nu}\right\}_{\nu \in \mathbb{N}}$ такая, что выполнено (1) $u$ $k_{\nu} \geqslant(1+\delta) / \ln 2 \cdot \ln n_{\nu}$, равна нулю.

2. О количественной возвращаемости. Пусть $X$ - метрическое пространство с метрикой $d(\cdot, \cdot)$ и сигма-алгеброй измеримых множеств $\Phi$, которая содержит все борелевские множества. Пусть также $T$ - измеримое, сохраняющее меру $\mu$ отображение $X$ в себя. Всюду ниже будем считать, что $\mu(X)=1$. Рассмотрим обычную меру Хаусдорфа $H_{s}(\cdot)$ на $X$. Будем говорить, что меры $\mu$ и $H_{s}$ согласованы, если любое $\mu$-измеримое множество является $H_{s}$-измеримым.

Следующую ниже теорему доказал М. Бошерницан в [3]. (Похожий результат независимо получил Н. Г. Мощевитин в [4].)

Теорема (Бошерницан). Пусть $X$ - метрическое пространство, $H_{s}$ и $\mu$ согласовань, для любого $\mu$-измеримого $A \mu(A)=H_{S}(A)$ и $T$ - отображение $X$ в себя, сохраняющее меру $\mu$. Тогда для почти всех $x \in X$ выполнено $\liminf _{n \rightarrow \infty}\left\{n^{1 / s} d\left(T^{n} x, x\right)\right\} \leqslant 1$.

3. О пространстве последовательностей. Как известно, преобразование Гаусса $T x=$ $\{1 / x\}, x \neq 0, T 0=0$, является левым сдвигом при разложении в цепную дробь и сохраняет меру $\nu(A)=1 / \ln 2 \int_{A} \frac{1}{1+x} d x$, которая эквивалентна мере Лебега.

Обозначим через $P_{k}$ отображение из пространства бесконечных цепных дробей в конечные цепные дроби $P_{k}\left(\left[a_{1}, a_{2}, \ldots\right]\right)=\left[a_{1}, \ldots, a_{k}\right]$. Интервалом $k$-го ранга $I_{k}=I_{k}\left(a_{1}, \ldots, a_{k}\right)$ называются все числа $\alpha$, у которых цепные дроби начинаются с $a_{1}, \ldots, a_{k}$. Рассмотрим неархимедову метрику на пространстве последовательностей $\left[a_{1}, a_{2}, \ldots\right]$ :

$$
D\left(\left[a_{1}, a_{2}, \ldots\right],\left[b_{1}, b_{2}, \ldots\right]\right)= \begin{cases}1, & \text { если } P_{1}\left(\left[a_{1}, a_{2}, \ldots\right]\right) \neq P_{1}\left(\left[b_{1}, b_{2}, \ldots\right]\right), \\ \nu\left(I_{k}\left(a_{1}, \ldots, a_{k}\right)\right) & \text { в противном случае }\end{cases}
$$

где $k \geqslant 1$ - максимальное такое, что $P_{k}\left(\left[a_{1}, a_{2}, \ldots\right]\right)=P_{k}\left(\left[b_{1}, b_{2}, \ldots\right]\right)$. 
Меры $H_{1}$ и $\nu$ согласованы. Достаточно проверить согласованность на $C_{n_{1}}, \ldots, n_{k}\left(A_{1}, \ldots, A_{k}\right)$ $=\left\{\alpha \mid a_{n_{1}} \in A_{1}, \ldots, a_{n_{k}} \in A_{k}\right\}$, где $A_{i} \subseteq \mathbb{N}$. Покрывая любой такой цилиндр $C$ элементарными цилиндрами $I_{k}\left(a_{1}, \ldots, a_{k}\right)$, убеждаемся, что $\nu(C) \leqslant H_{1}(C)$. Обратно, из определения метрики $D(\cdot, \cdot)$ следует, что любое покрытие $C$ может быть заменено на покрытие элементарными цилиндрами, откуда вытекает равенство мер $H_{1}$ и $\nu$.

4. Доказательство теоремы. 1) Так как $H_{1}$ и $\nu$ согласованы, то по теореме Бошерницана для почти всех $\alpha$ и, следовательно, для их соответствующих разложений в цепные дроби $\alpha=$ $\left[a_{1}, a_{2}, \ldots\right]$ существует последовательность номеров $\left\{n_{\nu}\right\}$ такая, что

$$
n_{\nu} D\left(\alpha, T^{n_{\nu}} \alpha\right) \leqslant 1 \text {. }
$$

По определению метрики $D(\cdot, \cdot) \quad D\left(\alpha, T^{n_{\nu}} \alpha\right)=\nu\left(I_{k}\right)$, где $k=k_{\nu}$ есть максимальное такое, что $a_{1}=a_{n_{\nu}+1}, a_{2}=a_{n_{\nu}+2}, \ldots, a_{k_{\nu}}=a_{n_{\nu}+k_{\nu}}$.

По известной теореме Хинчина-Леви [1] для любого $\varepsilon>0$ и для почти всех чисел $\alpha$ существует $M=M(\alpha)$, начиная с которого $q_{n}<(\gamma+\varepsilon)^{n}$. Так как $k_{\nu}$ возрастают, то можно считать $n_{1}$ настолько большим, что $k_{1}>M$. Тогда [1] $\nu\left(I_{k}\right)=O\left(\left|I_{k}\right|\right)$. Следовательно, по (2) $k_{\nu} \geqslant\left(6 \ln 2 / \pi^{2}-\varepsilon_{1}\right) \ln n_{\nu}$.

2) Следуя [1], обозначим через $M_{n}(x)$ меру множеств чисел $\alpha$ отрезка [0, 1], удовлетворяющих условиям $a_{1}=r_{1}, a_{2}=r_{2}, \ldots, a_{k}=r_{k} ; z_{k+n}<x$, где $z_{n+k}=\left[a_{n+k+1}, a_{n+k+2}, \ldots\right]$ и $0 \leqslant x \leqslant 1$. Тогда для выполнения условий

$$
a_{1}=r_{1}, a_{2}=r_{2}, \ldots, a_{k}=r_{k} ; a_{n+k+1}=r_{1}, a_{n+k+2}=r_{2}, \ldots, a_{n+2 k}=r_{k}
$$

необходимо и достаточно, чтобы $z_{k+n}$ принадлежал интервалу $k$-го ранга $I_{k}=I\left(r_{1}, \ldots, r_{k}\right)=$ $\left(\delta_{1}, \delta_{2}\right), \delta_{1}=p_{k} / q_{k}, \delta_{2}=\left(p_{k}+p_{k-1}\right) /\left(q_{k}+q_{k-1}\right)$, длины $1 /\left(q_{k}\left(q_{k}+q_{k-1}\right)\right)$. Обозначим множество всех чисел, удовлетворяющих (3), через $P_{n}\left(r_{1}, \ldots, r_{k}\right)$, тогда $\mu\left(P_{n}\left(r_{1}, \ldots, r_{k}\right)\right)=$ $\int_{\delta_{1}}^{\delta_{2}} M_{n}^{\prime}(x) d x$. Как известно [1; c. 101], последовательность функций $M_{0}^{\prime}(x), M_{1}^{\prime}(x), \ldots$ удовлетворяет функциональному уравнению Кузьмина и по [1; теорема 33$]$ мера $P_{n}\left(r_{1}, \ldots, r_{k}\right)$ есть

$$
\left|\frac{1}{\ln 2\left(q_{k}\left(q_{k}+q_{k-1}\right)\right)} \log \left(1 \pm \frac{\left|I_{k}\right|}{1+\frac{p_{k}}{q_{k}}}\right)\right|+O\left(\left|I_{k}\right| e^{-\lambda \sqrt{n}}\right) \ll\left|I_{k}\right|^{2}+\left|I_{k}\right| e^{-\lambda \sqrt{n}} .
$$

Рассмотрим множества $P_{N}$ тех $\alpha$, у которых

$$
a_{1}=a_{N+1}, a_{2}=a_{N+2}, \ldots, a_{K}=a_{N+K},
$$

и $K=\left[\log _{2} N^{1+\delta}\right]$, где $\delta>0$ - любое. Тогда множество $E=\bigcap_{M \geqslant 1} \bigcup_{N \geqslant M} P_{N}$ есть множество чисел, для которых бесконечно много раз выполняется (1). Оценим меру каждого $P_{N}$, $\mu\left(P_{N}\right)=\sum_{r_{1}, \ldots, r_{K}} \mu\left(P_{N-K}\left(r_{1}, \ldots, r_{k}\right)\right)$. Так как $\sum_{r_{1}, \ldots, r_{K}}\left|I_{K}\right|=1$, то по (4) $\mu\left(P_{N}\right) \ll$ $\sum_{r_{1}, \ldots, r_{K}}\left|I_{K}\right|^{2}+e^{-\lambda \sqrt{N-K}}$

Пусть $S_{k}=\sum_{r_{1}, \ldots, r_{k}}\left|I_{k}\right|^{2}$. Докажем, по индукции, что $S_{k} \leqslant(1 / 2)^{k}$. Для $S_{0}$ это очевидно. $S_{k}$ представляет собой сумму площадей квадратов со сторонами, равными длинам интервалов $k$-го ранга. Возьмем любой такой интервал $I_{k}=\left(p_{k} / q_{k},\left(p_{k}+p_{k-1}\right) /\left(q_{k}+q_{k-1}\right)\right)$. Он разбивается на интервалы $(k+1)$-го ранга $I_{k+1}^{1}, I_{k+1}^{2}, \ldots$, длины которых убьвают. $\left|I_{k+1}^{1}\right| /\left|I_{k}\right| \leqslant 1 / 2$, значит, $S_{k+1} \leqslant(1 / 2) \sum_{I_{k}}\left|I_{k}\right|^{2}=S_{k} / 2 \leqslant(1 / 2)^{k+1}$, что и требовалось. Таким образом,

$$
\sum_{N=1}^{\infty} \mu\left(P_{N}\right) \leqslant \sum_{N=1}^{\infty} \frac{1}{N^{1+\delta}}<\infty \text { и } \sum_{N=1}^{\infty} e^{-\lambda \sqrt{N-(1+\delta) \log _{2} N}}<\infty .
$$

Значит, по лемме Бореля-Кантелли мера множества $E$ равна нулю. Теорема доказана.

\section{СПИСОК ЛИТЕРАТУРЫ}

[1] А.Я. Хинчин. Цепные дроби. М.: Наука, 1978. [2] F. Spitzer. Principles of Random Walk. Princeton: Van Nostrand, 1964. [3] M. D. Boshernitzan // Invent. Math. 1993. V. 113. № 3. Р. 617-631. [4] Н. Г. Мошевитин // УМН. 1999. Т. 53. № 1. С. 223-224. 